\title{
Dengue fever triggering systemic lupus erythematosus and lupus nephritis: a case report
}

This article was published in the following Dove Press journal:

International Medical Case Reports Journal

30 October 2013

Number of times this article has been viewed

SH Talib

SR Bhattu

R Bhattu

SG Deshpande

DB Dahiphale

Department of Medicine and Nephrology, MGM Medical College and Hospital, Aurangabad, Maharashtra, India
Correspondence: SH Talib

Department of Medicine, MGM Medical College \& Hospital, N-6, Cidco, Aurangabad 431003, Maharashtra, India Email sftalib@gmail.com
Abstract: We report a rare case of dengue fever triggering systemic lupus erythematosus and lupus nephritis. The patient presented herself during a large outbreak of dengue fever in December 2012 in Maharashtra, India. The diagnosis of dengue fever was confirmed by the presence of NS-1 antigen during the first few days of febrile illness. Eight weeks later, kidney tissue biopsy studies revealed evidence of lupus nephritis on microscopic examination and immunofluorescence. The report interpreted it as focal proliferative glomerulonephritis and segmental sclerosis (Stage IIIC). The case was also found positive for perinuclear antineutrophil cytoplasmic antibodies by indirect immunofluorescence assay. An active and effective management of a case essentially calls for clear perception of differentiating dengue-induced lupus flare, antineutrophil cytoplasmic antibody-related nephropathy, and/or dengue-induced de-novo lupus disease. Dengue viremia may be the trigger for immune complex formation in patients who are predisposed to developing autoimmune diseases. The present case explains the importance of considering the diagnosis of dengue-related lupus nephritis as an atypical occurrence in appropriate situations, as in this case. It would not be improper to regard this escalating disease as an expanded feature of dengue.

Keywords: kidney biopsy, glomerulonephritis, segmental sclerosis, lupus flare, dengue viremia, autoimmune, de-novo lupus nephritis

\section{Introduction}

Dengue fever is a viral infection transmitted by mosquito Aedes aegypti found in the tropics and subtropics. Most symptomatic infections follow an uncomplicated course. Complications and unusual manifestations are now being increasingly recognized. Dengue disease and its severity is classified, based on the World Health Organization classification system 2011. ${ }^{1}$ There are four distinct subtypes of dengue virus. Infection with one serotype provides lifelong protective immunity to that serotype; however, there is no cross protectivity between serotypes. We encountered a case of lupus nephritis that occurred in later stages of dengue infection, and provide evidence that dengue alters the clinical disease beyond the acute phase of illness. Host factors are important in pathogenesis of lupus nephritis in dengue infection; the pathogenesis may be multifactorial and may result from a combination of pathogenic effects produced by the virus and immune responses of the host to the virus. Rajadhyaksha and Mehra from India in $2012^{2}$ reported the first ever case in world literature of dengue febrile illness evolving to lupus nephritis. We report yet another case of lupus nephritis observed post dengue febrile illness. 


\section{History}

The patient was a 32-year-old female who presented in December 2012 during a dengue epidemic, with history of high grade fever, cough, epistaxis, and melena for 5 days prior to hospitalization. Her fever was associated with headache, myalgias, and chills. She was perfectly healthy in the past and denied any significant history including that of renal disorders. On examination, the patient was moderately dyspneic, with respiratory rate of $30 /$ minute and was mildly febrile. Pulse rate was $48 \mathrm{bpm}$, which improved to $68-72 \mathrm{bpm}$ in sinus rhythm over the next 4 days. Her blood pressure was 120/80 mmHg. Clubbing, icterus, bleeding spots, and lymphadenopathy were not noted. Systemic examination revealed pneumonitis left base of lung. Laboratory investigations revealed the patient to be mildly anemic, thrombocytopenic, and with normal white blood cell count (Table 1). Chest X-ray and high resolution computed tomography showed evidence of pneumonitis in left lower lobe with reticulonodular infiltrates in left lung with bilateral minimal pleural effusion. Urine showed traces of protein; the blood and urine cultures were negative. Electrocardiography showed heart rate of $48 \mathrm{bpm}$ in sinus rhythm with QTc of 0.49 seconds. Serological tests for malaria, typhoid, HIV (human immunodeficiency virus), and hepatitis B and C were negative. Sputum for acid fast bacilli was also negative. Ultrasound abdomen showed non-tappable minimal ascites with mild hepatosplenomegaly. She was suspected of having dengue viral infection, the serologic test for dengue NS-1 antigen by enzyme-linked immunosorbent assay (ELISA) was positive, carried out on day 5 of febrile illness (first day of hospitalization). Dengue immunoglobulin $\mathrm{M}(\mathrm{IgM})$ and $\mathrm{IgG}$ antibodies were negative. She received supportive treatment with fluids and anti-pyretics. Her general condition improved after 10 days, and she was discharged on request with improved complete blood count. Subsequently, 4 weeks later, she again developed febrile illness and received symptomatic therapy by her family physician. Eight weeks post discharge from our hospital, she was re-hospitalized for her febrile illness, arthralgias of wrist, elbow, and knee

Table I Laboratory parameters during course of lupus nephritis development

\begin{tabular}{|c|c|c|c|c|}
\hline Parameters & Normal range & $\begin{array}{l}\text { Initial presentation with } \\
\text { dengue fever (before } \\
\text { lupus development) }\end{array}$ & $\begin{array}{l}\text { Development of } \\
\text { lupus nephritis } \\
\text { ( } 8 \text { weeks later) }\end{array}$ & $\begin{array}{l}\text { I0-week follow-up - } \\
\text { post lupus therapy }\end{array}$ \\
\hline Hemoglobin (gm/dL) & $12-16$ & 9.9 & 10.0 & 14.3 \\
\hline Leucocyte count $\left(/ \mathrm{mm}^{3}\right)$ & $4,000-10,500$ & 6,060 & 9,270 & 11,100 \\
\hline Platelet count $\left(\mathrm{lac} / \mathrm{mm}^{3}\right)$ & $1.5-4.5$ & 0.60 & 3.98 & 3.34 \\
\hline Hematocrit (\%) & $37-50$ & 32.3 & 29.9 & 32 \\
\hline ESR & Up to 20 & 20 & 74 & 38 \\
\hline Urea (mg \%) & $15-35$ & 16 & 18 & 32 \\
\hline Serum creatinine (mg \%) & $0.6-1.3$ & 1.0 & 0.9 & 0.8 \\
\hline Serum sodium (mE/L) & $135-145$ & 133 & 134 & 140 \\
\hline Serum potassium (mE/L) & $3.5-5.5$ & 3.8 & 4.1 & 3.6 \\
\hline SGPT (U/L) & $5-55$ & 25 & 24 & 51 \\
\hline SGOT (U/L) & $5-55$ & 35 & 33 & 23 \\
\hline Total protein (gm \%) & $6.2-8.4$ & 6.9 & 6.5 & 7.0 \\
\hline Albumin (gm \%) & $3.5-5.0$ & 2.5 & 2.6 & 3.8 \\
\hline Globulin (gm/dL) & $2-4$ & 4.4 & 4.4 & 3.2 \\
\hline CPK total (IU/L) & $60-400$ & Not done & Not done & 51 \\
\hline Urine microscopy & $\begin{array}{l}\text { Absent proteins, } \\
\text { cells, casts }\end{array}$ & Trace proteins, no casts & $\begin{array}{l}3+\text { proteins, } \\
2-3 \text { RBCs/HPF, no casts }\end{array}$ & $\begin{array}{l}\text { Trace proteins, } \\
\text { no casts }\end{array}$ \\
\hline 24 -hour urinary protein (mg/24h) & $<300$ & Not done & 1,130 & 422 \\
\hline ANA (immunofluorescence) & - & Not done & $\begin{array}{l}\text { Positive (homogenous) } \\
\text { I:2,560 }\end{array}$ & $\begin{array}{l}\text { Positive (homogenous) } \\
\text { I:80 }\end{array}$ \\
\hline Anti-dsDNA (immunofluorescence) & - & Not done & Positive I:80 & Negative \\
\hline Complement C3 (mg/dL) & $90-180$ & Not done & 105 & 76.2 \\
\hline Complement C4 (mg/dL) & $10-40$ & Not done & 18 & 15.8 \\
\hline Coombs test (direct and indirect) & Negative & Not done & Negative & Negative \\
\hline P-ANCA (IIF) & - & Not done & Strongly positive $1: 320$ & Weakly positive 1:80 \\
\hline $\begin{array}{l}\text { IHC, ISH - dengue RNA virus } \\
\text { in tissue biopsy }\end{array}$ & - & Not done & Negative & \\
\hline
\end{tabular}

Abbreviations: ANA, antinuclear antibody; Anti-dsDNA, anti-double-stranded deoxyribonucleic acid; CPK, creatine phosphokinase; ESR, erythrocyte sedimentation rate; HPF, high power field; IHC, immunohistochemistry; IIF, indirect immunofluorescence; ISH, in situ hybridization; p-ANCA, perinuclear antineutrophil cytoplasmic antibody; SGOT, serum glutamic oxaloacetic transaminase; SGPT, serum glutamic pyruvic transferase; RBC, red blood cell. 
joints and developing pedal edema. Laboratory investigations showed $3+$ proteinuria (1,130 mg per 24 hours) and serum creatinine of $0.9 \mathrm{mg} / \mathrm{dL}$. Systemic lupus erythematosus with active lupus nephritis was suspected. Antinuclear antibody was positive with homogenous pattern $(1: 2,560)$, anti-dsDNA (anti-double-stranded deoxyribonucleic acid) titers were also positive (1:160), and the patient fulfilled 4/11 American College of Rheumatology criteria for systemic lupus nephritis. Rheumatoid factor and Coombs test were negative. Perinuclear antineutrophil cytoplasmic antibodies (p-ANCA) test was reported strongly positive $(1: 320, \mathrm{~N}=1: 20)$ by indirect immunofluorescence (IIF). p-ANCA test by ELISA was not done. Light microscopy, electron microscopy, and immunofluorescence studies on the renal tissue biopsy revealed evidence of focal proliferative lupus glomerulonephritis, ISN/ RPS (International Society of Nephrology/Renal Pathology Society) class IIIC with mild activity and minimal chronicity. The details of microscopic description of light microscopy, immunofluorescence, and electronic microscopy, along with images, are attached separately in the Supplementary material. On request, immunohistochemistry for dengue virus RNA (ribonucleic acid) complex on tissue biopsy was carried out at University of Malaya, Malaysia. The report was however negative. The patient was treated with infusion of methylprednisolone ( $1 \mathrm{~g}$ ) for 5 days and then oral prednisolone ( $60 \mathrm{mg}$ /day) for 2 months, followed by tapering off (2.5 mg fortnightly) along with mycophenolate mofetil (1,500 mg/day), with advice to continue for 6 months with supportive therapies. She showed gradual improvement in 2 weeks and was discharged. Six weeks post discharge, her laboratory parameters were: hemoglobin $12.0 \mathrm{~g} / \mathrm{dL}$, platelets $307,000 / \mathrm{mm}^{3}$, serum creatinine $0.7 \mathrm{mg} / \mathrm{dL}$, total urinary proteins declined to $376 \mathrm{mg} /$ day, and other investigations as shown in Table 1. The patient was comfortable and stable. Six-weekly periodic follow-up was suggested for 1 year.

\section{Discussion}

The dengue virus has significant impact on the health of those it affects and represents a burdensome cost to the patient in situations where health infrastructure is not adequately defined. Furthermore, the disease occurs with atypical presentations and varied threats.

The diagnosis of dengue is primarily a clinical one, serological tests are confirmative and require cautious interpretation, particularly with secondary dengue. The dengue IgM antibody has a high positive predictive value in endemic regions and one should be aware of its limitations. The case under discussion presented to us during an outbreak of dengue fever that hit the state of Maharashtra, India, in November and December 2012.

There have been scanty reports in the literature regarding the inter-relationship of dengue with lupus nephritis and systemic lupus erythematosus. de Souza and de Moura in 2010, described a case of dengue fever in a known case of systemic lupus erythematosus mimicking lupus flare. ${ }^{3} \mathrm{~A}$ case is also reported of cardiac tamponade in a patient with dengue fever and lupus nephritis. ${ }^{4}$ Santosa et al in $2012^{5}$ proposed a diagnostic algorithm to guide diagnosis of dengue infection in circumstances of atypical disease manifestation or progression and further suggested that dengue NS-1 antigen can be detected with $92 \%$ sensitivity and $100 \%$ specificity when the test is done within 9 days of illness.

Our case findings, however, project three main possibilities pertaining to association of dengue fever with lupus nephritis:

1. The case could be related to p-ANCA-positive nephropathy.

2. The case had subclinical lupus syndrome with lupus nephritis and subsequent occurrence of dengue resulted in lupus flare-up.

3. The occurrence of lupus nephritis is a de-novo disease noted post-dengue viral illness.

Lupus nephritis is described as classic immune complex mediated renal disease. Approximately $20 \%$ of patients with systemic lupus erythematosus have p-ANCA positivity by IIF. Seropositivity by ELISA is less frequent, and the target antigens are most commonly lactoferrin, cathepsin-G, and myeloperoxidase. ${ }^{6}$ ANCA positivity by IIF in patients with systemic lupus erythematosus is greater than ELISA. This is due to the presence of non-proteinase 3, non-myeloperoxidase ANCA, as well as reasons attributed to the difficulties in distinguishing p-ANCA from antinuclear antibody by IIF assay. ${ }^{7}$ In our case p-ANCA was positive by IIF assay in high titer. However, ELISA test was not done. Nasr et al in $2008^{7}$ in a small cohort study supported the pathogenic role of ANCA in a small group of systemic lupus erythematosus, wherein renal biopsies reveal extensive necrosis and crescent formations in the absence of significant endocapillary proliferation of subendothelial deposits, as opposed to the present case wherein microscopic and immunofluorescence studies were interpreted as focal proliferative glomerulonephritis with segmental sclerosis.

The other possibility - that the case had subclinical syndrome with lupus nephritis, and subsequent occurrence of dengue resulted in lupus flare - is stronger in this case. The serum at the time of dengue fever was not stored, and hence 
autoantibody tests were not undertaken later. These tests could have helped to understand lupus interrelationship with dengue more clearly, as the two diseases have similar clinical manifestations.

Furthermore, viruses and bacterial infections are known to trigger lupus activity. Viruses like cytomegalovirus and Epstein-Barr virus can augment the immune complex in systemic lupus erythematosus. ${ }^{8,9}$ RNA dengue-virus-detection by in-situ hybridization was requested in the kidney tissue block of this lupus nephritis case, and the result was negative. Jessie et al in $2004^{10}$ from Malaysia described certain seropositive cases of dengue showing negativity in a kidney tissue specimen and argued the possibility that whatever RNA of replicative intermediates was present in the tissue specimen might have been destroyed as a result of formalin-fixation and tissue processing.

The other possibility that remains is that the dengue may result in, or alter the course of, clinical disease beyond acute phase of dengue illness, evolving to lupus nephritis as noted in the present case. It would be difficult to believe whether the disease is de-novo or lupus flare. However, simultaneity or concomitant presentation of both disorders can also not be denied in the present case.

\section{Conclusion}

Dengue febrile illness may manifest itself with atypical presentations occurring beyond the acute phase of illness. Lupus nephritis is one such rare presentation which may occur as a de-novo disease or as lupus flare. In situations of this sort, therefore, it seems advisable to extend investigations for recognition of lupus disease in all sero-positive dengue fever, especially in dengue endemic regions, to avoid undesired delayed diagnosis of systemic lupus erythematosus/nephritis and its management.

\section{Disclosure}

No sources of funding were used to assist in preparation of this case report, and the authors report no conflicts of interest in this work.

\section{References}

1. World Health Organization, Regional Office for South-East Asia. Comprehensive Guidelines for Prevention and Control of Dengue and Dengue Hemorrhagic Fever. Revised and Expanded Edition. SEARO Technical Publication Series No 60, New Delhi, India: 2011; chapter 4:26-27.

2. Rajadhyaksha A, Mehra S. Dengue fever evolving into systemic lupus erythematosus and lupus nephritis: a case report. Lupus. 2012;9: 999-1002.

3. de Souza SP, de Moura CG. Dengue mimicking a lupus flare. J Clin Rheumatol. 2010;16:47-48.

4. Kumar S, Iuga A, A Jean R. Cardiac tamponade in a patient with dengue fever and lupus nephritis: a case report. J Intensive Care Med. 2010;25:175-178.

5. Santosa A, Poh Z, Teng G. Delayed diagnosis of systemic lupus erythematosus due to misinterpretation of dengue serology. Scand $J$ Rheumatol. 2012;41:77-79.

6. Sen D, Isenberg DA. Antineutrophil cytoplasmic autoantibodies in systemic lupus erythematosus. Lupus. 2003;12:651-658.

7. Nasr SH, D'Agati VD, Park HR, et al. Necrotizing and crescentic lupus nephritis with antineutrophil cytoplasmic antibody seropositivity. Clin J Am Soc Nephrol. 2008;3(3):682-690.

8. Chen CJ, Lin KH, Lin SC, et al. High prevalence of immunoglobulin A antibody against Epstein-Barr virus capsid antigen in adult patients with lupus with disease flare: case control studies. J Rheumatol. 2005;32: $44-47$.

9. Su BY, Su CY, Yu SF, Chen CJ. Incidental discovery of high systemic lupus erythematosus disease activity associated with cytomegalovirus viral activity. Med Microbial Immunol. 2007;196:165-170.

10. Jessie K, Fong MY, Devi S, Lam SK, Wong KT. Localization of dengue virus in naturally infected human tissues by immunohistochemistry and in situ hybridization. J Infect Dis. 2004;189:1411-1418. 


\section{Supplementary material}

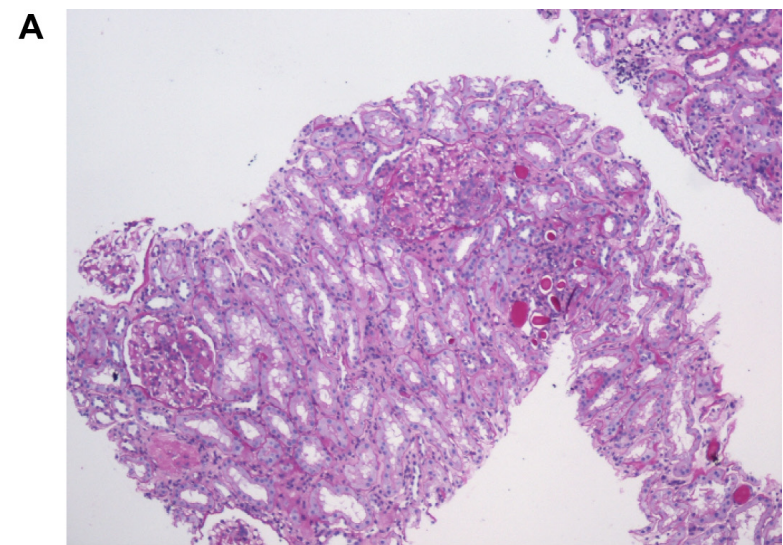

B

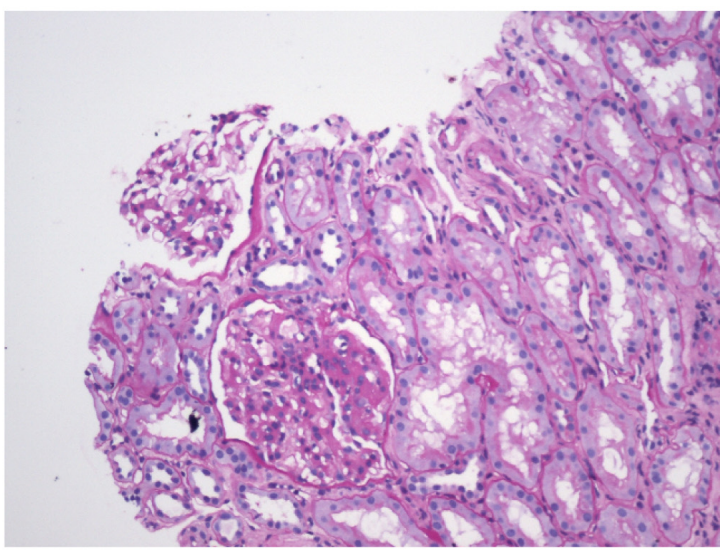

C

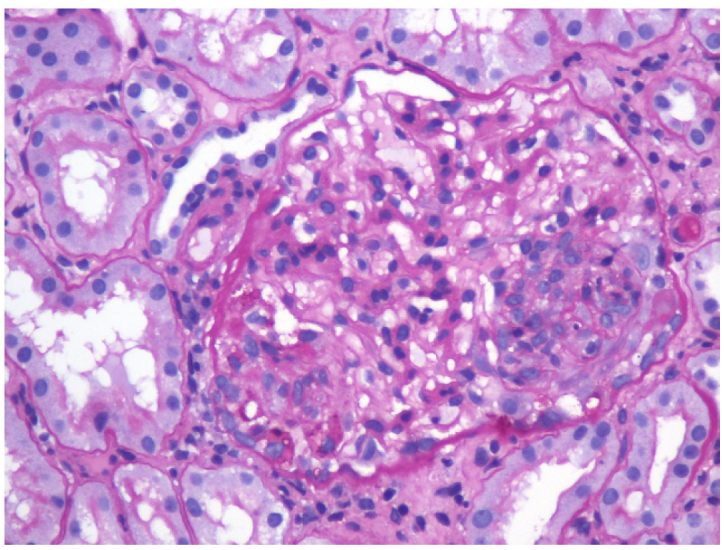

Figure SI Images from light microscopy, electron microscopy, and immunofluorescence studies on the renal tissue biopsy. (A) Low power view showing three glomeruli, two with segmental lesions (PASXI00), (B) segmental sclerosis in one glomerulua (PASX400), and (C) segmental endocapillary proliferation obliterating the capillary lumina (PASX400). Notes: Final diagnosis performed by Stephen M Bonsib, MD Nephropath, Arkansas, USA. Pathology No: SI3-02352. Specimen submitted by Swarnalata G, MD, for kidney, consult biopsy.

\section{Microscopic description Light microscopy}

PAS stained sections contain renal cortex and medulla. Six glomeruli are present, one is sclerotic. One glomerulus shows segmental endocapillary proliferation and one glomerulus shows segmental sclerosis.

\section{Immunofluorescence}

The IF studies show five glomeruli with significant deposits of all immunoglobulins, $\mathrm{C} 3$ and $\mathrm{C} 1 \mathrm{q}$, in a predominant mesengial pattern.

\section{Electron microscopy}

Ultrastructural examination shows loss of podocyte foot processes. The capillary loops are open with normal basement membranes. There is an occasional subepithelial deposit and a rare small subendothelial deposit. The mesangium is expanded and contains numerous very electron dense deposits.
International Medical Case Reports Journal

\section{Publish your work in this journal}

The International Medical Case Reports Journal is an international, peer-reviewed open-access journal publishing original case reports from all medical specialties. Previously unpublished medical posters are also accepted relating to any area of clinical or preclinical science. Submissions should not normally exceed 2,000 words or

\section{Dovepress}

4 published pages including figures, diagrams and references. The manuscript management system is completely online and includes a very quick and fair peer-review system, which is all easy to use. Visit http://www.dovepress.com/testimonials.php to read real quotes from published authors. 